\title{
Professionelle Urteilsbildung in der Sozialen Arbeit
}

\section{Anforderungen und Herausforderungen}

In diesem Schwerpunkt widmen sich die Autor_innen dem komplexen und anspruchsvollen Prozess der Urteilsbildung in differenten Feldern der Sozialen Arbeit. Obgleich das Fällen fachlicher Urteile und die damit verknüpften Entscheidungen ein zentrales Charakteristikum professioneller Praxis ist, wird der praktische Prozess des Vollzugs der Urteilsbildung noch immer allzu selten in den Blick genommen. Dabei hängt doch gerade die Handlungsfähigkeit der Fachkräfte, wollen sie ihrer Aufgabe einer hinreichend gelingenden Gestaltung professioneller Bildungs-, Beratungs-, Begleitungs-, Unterstützungs- und Hilfeprozesse gerecht werden, wesentlich von ihrer Befähigung und Fähigkeit ab, trotz hoher Komplexität, unklarer Informationslage und unzureichender Ressourcen äußerst schnell Entscheidungen zu treffen.

$\mathrm{D}$ araus ergibt sich ein hohes Maß an Ungewissheit im Hinblick auf die zu wählende Handlungsstrategie; Fachkräfte befinden sich oft in hochkomplexen Situationen, die durch Unvorhersehbarkeit, Widersprüchlichkeit, konfliktreiche Zielvorstellungen, aber auch durch unterschiedliche Annahmen und Erwartungen, gekennzeichnet sind (vgl. Dewe und Otto 2011). Das heißt, die typischerweise labilen, offenen und diffusen Situationen sind durch eine begrenzte Durchschaubarkeit und Planbarkeit geprägt und im
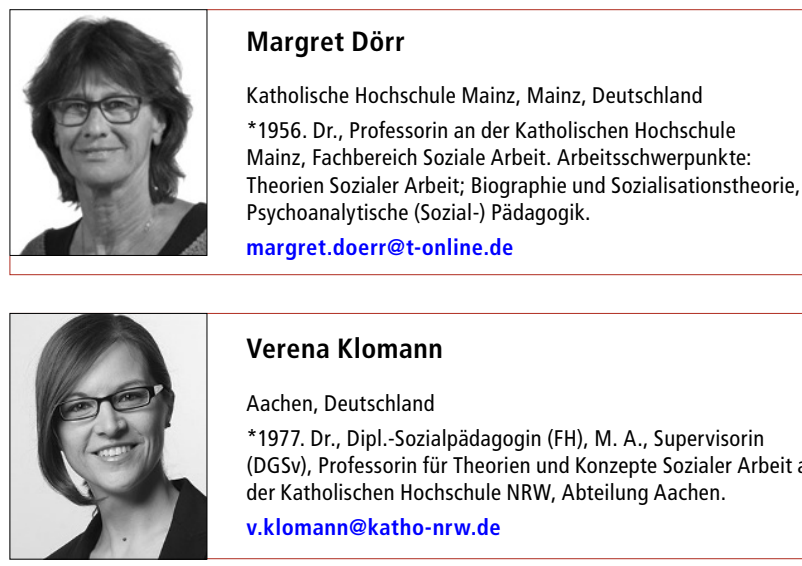

\section{Verena Klomann}

Aachen, Deutschland

*1977. Dr., Dipl.-Sozialpädagogin (FH), M. A., Supervisorin (DGSv), Professorin für Theorien und Konzepte Sozialer Arbeit an der Katholischen Hochschule NRW, Abteilung Aachen.

v.klomann@katho-nrw.de

Zusammenfassung Urteilsbildung in der Sozialen Arbeit ist anspruchsvoll und komplex. Trotzdem ist der Prozess der Urteilsbildung bisher selten Gegenstand wissenschaftlicher Betrachtungen. Der Beitrag führt in den Schwerpunkt „Professionelle Urteilsbildung in der Sozialen Arbeit“ ein und stellt die einzelnen Beiträge kurz vor.

Schlüsselwörter Urteilsbildung, Widersprüche, Uno-actuPrinzip, Fallkonstruktion
Hinblick auf ihre Entwicklung nur gering vorhersehbar (vgl. Combe und Helsper 2004). Diese, für die Soziale Arbeit typische, Handlungsstruktur ermöglicht es nicht, professionelles Handeln entlang einheitlicher Handlungsregeln zu gestalten - sie kann daher nicht auf der Basis eines rein kognitiven Abwägens von Informationen erfolgen. Vielmehr gilt es, einen kompetenten Umgang mit einer auf Ungewissheit basierten Handlungsstruktur zu entwickeln.

Zudem ist die Soziale Arbeit in besonderer Weise durch das uno-actu Prinzip gekennzeichnet: Die Produktion und die Konsumtion der Dienstleistung finden am selben Ort und zur selben Zeit in derselben Handlung statt. Produktions- und Konsumtionsprozess fallen also zeitlich zusammen und erfordern die Anwesenheit und Mitwirkungsbereitschaft der Adressat_innen (vgl. Schaarschuch 1996; Kessl und Otto 2011). Daher bedarf die Erzeugung einer hinreichend angemessenen Urteilsbildung der Befähigung und Fähigkeit von Professionellen in der Sozialen Arbeit, wissenschaftliches Wissen und praktisches Handlungswissen sowohl zu kontextualisieren als auch beide Wissenstypen zu relationieren (vgl. Dewe und Otto 2011), und dies zugleich verbunden mit dem Vermögen eines reflexiven Umgangs mit Wissen und Nicht-Wissen (vgl. Dewe 2009).

Dies sind hohe und zugleich störanfällige Anforderungen. Denn obgleich professionelle Autonomie sowie fachliche Handlungs- und Entscheidungsspielräume von zentraler Bedeutung sind, so weisen empirische Studien nach, wie sehr die Eigenlogiken von Organisationen das Handeln der Fachkräfte zum Nachteil der Adressat_innen beeinflussen oder organisationale Handlungsvorgaben, wie z. B. Verfahrensstandards bei 


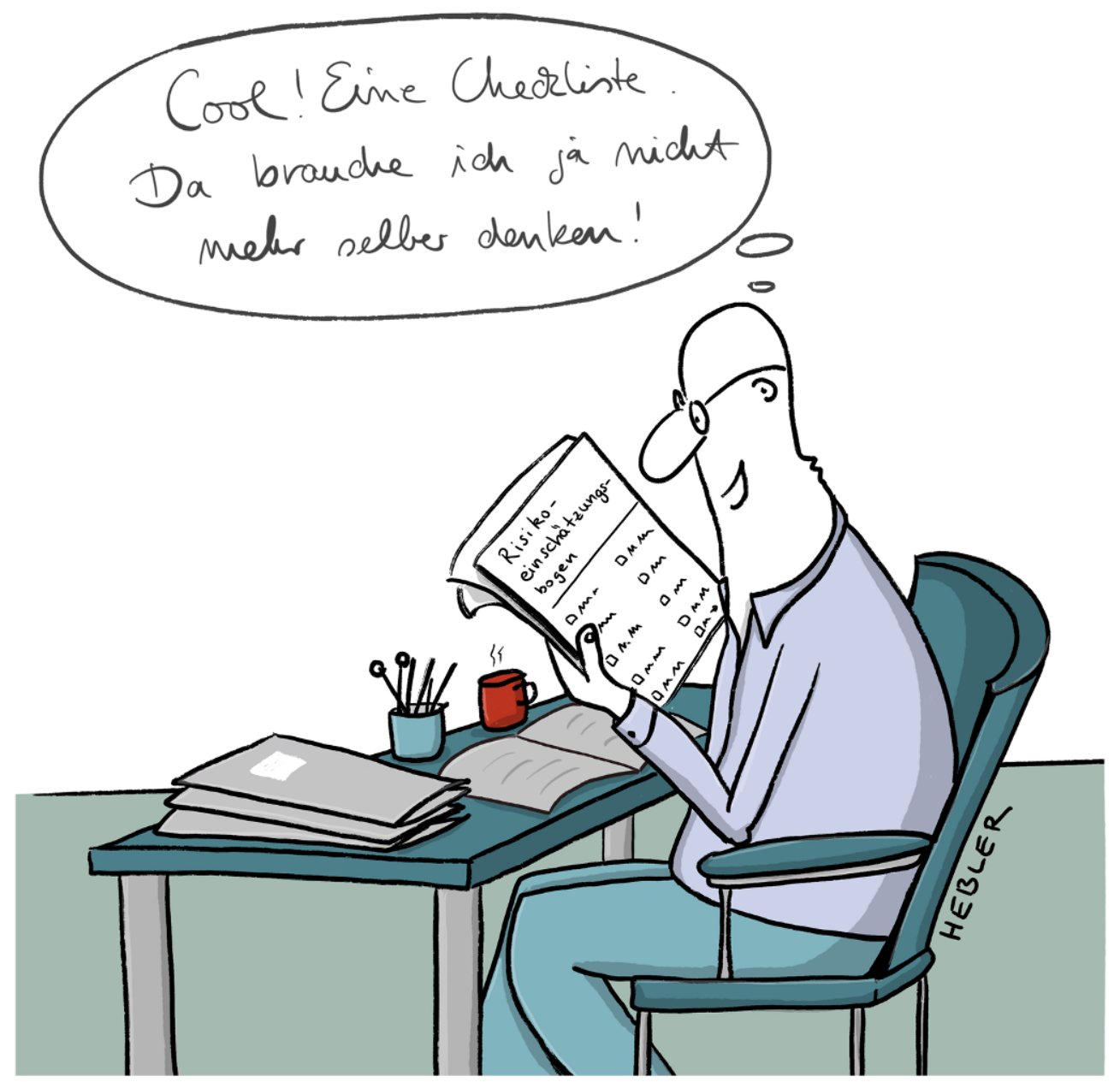

(c) Ruth Hebler

Jugendämtern, professionelle Urteile vorstrukturieren (vgl. Bauer 2010), dies verbunden mit der Gefahr einer Perpetuierung von Hilfekarrieren auf Seiten der Adressat_innen (vgl. Hamberger 2008). Zudem werden Prozesse der Fallkonstruktion, mithin der Urteilsbildung, nicht nur durch mehr oder weniger durchschaute interund intra-institutionelle Bedingungen und Kommunikationsstrukturen (mit) gesteuert, auch sozialstrukturelle Bedingungen wie z. B. soziodemographische Besonderheiten (vgl. Petrat und Santen 2010) oder das (Nicht) Vorhandensein von Angeboten beeinflussen die Hilfeentscheidungen der Fachkräfte wirkmächtig (Schrapper und Spies 2003).

Gleichzeitig ist dieser Prozess besonders anfällig für expertokratische und paternalistische Ausformungen - die insbesondere im Zuge neoliberaler Entwicklungen und veränderter Gerechtigkeitsvorstellungen - an Selbstverständlichkeit zu gewinnen scheinen. Entsprechend wollen wir - hieran anknüpfend - Anforderungen und Herausforderungen professioneller Urteilsbildung in der Sozialen Arbeit grundsätzlich und entlang ausgewählter Arbeitsfelder diskutierten und hierdurch zur Reflexion und Weiterentwicklung von professioneller(n)
Urteilsbildung(-sprozessen) als zentraler Modus professioneller Sozialer Arbeit beitragen.

\section{Die Beiträge des Schwerpunkts}

Stephan Wolff wirft in seinem Artikel einen Blick zurück auf seine ethnomethodologische Studie Die Produktion von Fürsorglichkeit (1983). Dabei plädiert er für seine damals wie aktuell favorisierte Perspektive, die Praxis nicht defizitorientiert, weil mehr oder weniger von idealen Modellen oder Vorgaben abweichend, zu betrachten. Vielmehr sei von der heuristischen (!) Unterstellung auszugehen, dass die jeweilige Praxis unter den gegebenen Bedingungen eine eigene Rationalität entwickelt, die als Hinweis zu lesen ist, wie anstehende Arbeitsprobleme angesichts praktischer Umstände mit einer dafür charakteristischen Form von Urteilsbildung und daraus folgenden Weisen ,kompetenten Handelns“ angemessen bearbeitet werden. Erst im zweiten Schritt seien diese auf Nebenfolgen und funktionale Äquivalente hin kritisch zu prüfen.

Holger Ziegler arbeitet in seinem Beitrag Paternalismus und Citizenship. Herausforderungen professioneller Urteilsbildung im Sozialinvestionsstaat das Pater- 


\section{Extrablick: Urteilsbildung in der Sozialen Arbeit}

nalismusproblem als ein Kernproblem professioneller Urteilsbildung als Grundlage einer angemessenen Praxis heraus, das sich mit einer zunehmenden sozialpolitischen Bedeutung Sozialer Arbeit verschärft. Die (normative) Ausrichtung des wohlfahrtsstaatlichen Arrangements prägt das Paternalismusproblem, wobei hier eine Verschiebung in Richtung eines sozialinvestionsstaatlichen Begründungsrahmens deutlich wird. Hiermit geht eine veränderte Relevanz sozialpädagogischer Wohlfahrtsleistungen einher, was wiederum die Auseinandersetzung mit dem professionellen Umgang hiermit beziehungsweise hieraus resultierenden Entwicklungsbedarfen betont.

Sabine Ader fragt in ihrem Beitrag Analytischer ,Scharfsinn' und geschulte Intuition im Dialog. Fallverstehen und sozialpädagogische Diagnostik als Schlüsselprozess professioneller Urteilsbildung nach den Anforderungen an Professionelle in der Sozialen Arbeit - insbesondere in der Einzelfallarbeit im Kontext der Kinder- und Jugendhilfe -, wenn sie ihrer Aufgabe gerecht werden wollen, trotz Ungewissheitshorizont und häufig unter zeitlichem Druck, zu fachlich fundierten Urteilen und Entscheidungen zu gelangen. Dabei umreißt sie u. a. die Grundbewegung für Erkenntnisgewinn und hebt das Erfordernis der Gleichzeitigkeit von Theorie- und Fallverstehen, begleitet von einer grundsätzlichen Diskursbereitschaft, hervor. Einen besonderen Blick widmet sie der für eine reflexive Professionalität nur unzureichend beachteten Notwendigkeit, professionelles Wissen und - bereits im Studium zu schulende - Intuition fortwährend im Dialog zu halten.

In ihrem Beitrag Vom Problem mit der Bestimmung des Fallproblems. Herausforderungen bei der interaktiven Fallkonstitution als ein Kernprozess professioneller Praxis im Kontext (möglicher) Kindeswohlgefährdungen veranschaulicht Cornelia Rüegger am Beispiel einiger Ergebnisse ihrer qualitativ-empirischen Studie, warum die Verknüpfung verschiedener Wissensbestände zur Bestimmung einer Fallproblematik nicht trivial ist. Sie zeigt auf, wie Urteilsbildungen durch je spezifische Ausgangslagen, organisational formierte Interaktionsordnungen sowie weitere Präkonstruktionen bereits vor jeglicher realen Begegnung zwischen Fachkraft und Adressat_in folgenreich beeinflusst werden. Zudem erläutert sie kommunikative Bearbeitungsanforderungen bei Gesprächseröffnungen sowie im Prozess der Problemexploration mit Adressat_innen und thematisiert das (Fallen-) Potenzial defensiver Praktiken während der Interaktionsverläufe zur Einschätzung einer Gefährdung des Kindeswohls.

Hannes Käckmeister skizziert in seinem Beitrag Umgang mit Überkomplexität. Professionelle Urteilsbil- dung im Rabmen der Alterseinschätzung junger Geflüchteter die Komplexität von Alterseinschätzungen und den hiermit einhergehenden vielfältigen Herausforderungen. Aufbauend auf empirischem Datenmaterial arbeitet er heraus, in welche Dilemmata sowohl Fachkräfte als auch die jungen Menschen, deren Alter es einzuschätzen gilt, in diesem Prozess geraten und dass diese nicht aufzulösen, sondern lediglich (mühsam) auszubalancieren sind. Er stellt weiter anschaulich dar, welche Fallstricke mit der unterlegten Prozessstruktur des Misstrauens einhergehen, wie Fachkräfte mit Widersprüchen umzugehen versuchen, mit welchen unerwünschten $\mathrm{Ne}$ benfolgen eine qualifizierte Inaugenscheinnahme verbunden ist und welche kreativen Strategien die Sozialarbeitenden in „Zweifelsfällen“ entwickeln.

Im Mittelpunkt des Beitrags von Norbert FrietersReermann und Susanne Bücken, Kritisch-reflexive Urteilskompetenz in der Bildungsarbeit - Konfliktsensible und rassismuskritische Perspektiven, stehen Bildungsprozesse, die von den Autor_innen als niemals neutral und - in der hochkomplexen diversifizierten Weltgesellschaft - als extrem konfliktanfällig und spannungsreich eingeordnet werden. Hiermit einhergehend ist zu konstatieren, dass Bildungsangebote, -strukturen, -formate und -materialien oftmals ungewollt dazu beitragen (können), dass vorhandene Konfliktpotentiale verstärkt, Diskriminierungstendenzen verschärft und Exklusionsdynamiken erhöht werden. In der Folge wird die Notwendigkeit einer umfassenden kritisch-reflexiven Urteilskompetenz aller verantwortlichen Akteur_innen aus einer konfliktsensiblen und rassismuskritischen Bildungsperspektive herausgearbeitet.

Auch Mario Rund betrachtet in seinem Beitrag „Kontingente Bauchgefüble. Urteilen und Entscheiden in der Sozialraumarbeit im Kontext von Programmierungen und ökonomisierter Wissensproduktion" ein überaus heterogenes und komplexes Arbeitsfeld. Auf der Folie des Konzepts der „Anrufung“ (Althusser 1977) akzentuiert er die Relevanz der professionellen Subjektivierung für Urteilsbildungsprozesse. Der Autor zeigt, in welcher Weise Förderprogramme als eine grundsätzliche Einflussgröße auf die Konstitution von Professionssubjekten zu betrachten sind und entziffert de-professionelle Folgen für die fachliche Urteilsbildung von Fachkräften, die durch eine um sich greifende beratungswirtschaftliche Produktionslogik von Programmen präformiert werden. Hierauf aufbauend ergibt sich die Notwendigkeit, diese Einsichten in die Reflexion eigener Entscheidungs- und Urteilspraktiken einzubeziehen. 
Funding. Open Access funding enabled and organized by Projekt DEAL.

Open Access. Dieser Artikel wird unter der Creative Commons Namensnennung 4.0 International Lizenz veröffentlicht, welche die Nutzung, Vervielfältigung, Bearbeitung, Verbreitung und Wiedergabe in jeglichem Medium und Format erlaubt, sofern Sie den/die ursprünglichen Autor(en) und die Quelle ordnungsgemäß nennen, einen Link zur Creative Commons Lizenz beifügen und angeben, ob Änderungen vorgenommen wurden.

Die in diesem Artikel enthaltenen Bilder und sonstiges Drittmaterial unterliegen ebenfalls der genannten Creative Commons Lizenz, sofern sich aus der Abbildungslegende nichts anderes ergibt. Sofern das betreffende Material nicht unter der genannten Creative Commons Lizenz steht und die betreffende Handlung nicht nach gesetzlichen Vorschriften erlaubt ist, ist für die oben aufgeführten Weiterverwendungen des Materials die Einwilligung des jeweiligen Rechteinhabers einzuholen.

Weitere Details zur Lizenz entnehmen Sie bitte der Lizenzinformation auf http://creativecommons.org/licenses/by/4.0/deed.de.

\section{Literatur}

Althusser, L. (1977). Ideologie und ideologische Staatsapparate. In L. Althusser (Hrsg.), Ideologie und ideologische Staatsapparate (S. 108-153). Hamburg, Berlin: VSA.

Bauer, P. (2010). Organisatorische Bedingungen der Fallkonstitution in der Sozialen Arbeit. Zeitschrift für Pädagogik, 56(2), 249-266.

Combe, A., \& Helsper, W. (2004). Professionalität. In H.-U. Otto, T. Rauschenbach \& P. Vogel (Hrsg.), Erziehungswissenschaft: Professionalität und Kompetenz (S. 29-47). Opladen: Leske + Budrich.

Dewe, B. (2009). Reflexive Professionalität: Maßgabe für Wissenstransfer und Theorie-Praxis-Relationierung im Studium der Sozialarbeit. In A. Riegler, S. Hojnik \& K. Posch (Hrsg.), Soziale Arbeit zwischen Profession und Wissenschaft. Vermittlungsmöglichkeiten in der Fachhochschulausbildung (S. 47-63). Wiesbaden: VS.

Dewe, B., \& Otto, H.-U. (2011). Professionalität. In H.-U. Otto \& H. Thiersch (Hrsg.), Handbuch Soziale Arbeit (4. Aufl. S. 1143-1153). München, Basel: Reinhardt Verlag.

Hamberger, M. (2008). Erziehungshilfekarrieren. Belastende Lebensgeschichte und professionelle Weichenstellungen. Frankfurt a. M.: IGFH Internationale Gesellschaft für erzieherische Hilfen.

Kessl, F., \& Otto, H.-U. (2011). Soziale Arbeit und soziale Dienste. In A. Evers, R. Heinze \& T. Olk (Hrsg.), Handbuch Soziale Dienste (S. 389-403). Wiesbaden: VS.

Petrat, A., \& Santen, E. (2010). Does help help? Zeitschrift für Erziehungswissenschaft, 13(2), 249-271.

Schaarschuch, A. (1996). Dienst-Leistung und Soziale Arbeit. Theoretische Überlegungen zur Rekonstruktion Sozialer Arbeit als Dienstleistung. Widersprüche. Zeitschrift für sozialistische Politik im Bildungs-, Gesundheitsund Sozialbereich, 16(59), 87-97.

Schrapper, C., \& Spies, S. (2003). Hilfeplanung als Kontraktmanagement? Erster Zwischenbericht des Forschungs- und Entwicklungsprojekts „Hilfeplanung als Kontraktmanagement?". München: Deutsches Jugendinstitut.

Hier steht eine Anzeige. Springer 